\title{
Editorial
}

\section{New Developments in Fluid Mechanics and Its Engineering Applications}

\author{
Oluwole Daniel Makinde, ${ }^{1}$ Waqar Ahmed Khan, ${ }^{2}$ and Tirivanhu Chinyoka ${ }^{3}$ \\ ${ }^{1}$ Faculty of Military Science, Stellenbosch University, Private Bag X2, Saldanha 7395, South Africa \\ ${ }^{2}$ Department of Engineering Sciences, Pakistan Navy Engineering College, National University of Sciences \& Technology, \\ Karachi 75350, Pakistan \\ ${ }^{3}$ Center for Research in Computational and Applied Mechanics, University of Cape Town, Rondebosch 7701, South Africa \\ Correspondence should be addressed to Oluwole Daniel Makinde; makinded@gmail.com
}

Received 8 December 2013; Accepted 8 December 2013

Copyright (C) 2013 Oluwole Daniel Makinde et al. This is an open access article distributed under the Creative Commons Attribution License, which permits unrestricted use, distribution, and reproduction in any medium, provided the original work is properly cited.

Fluid mechanics is an active field of research with many unsolved or partially solved problems in science and engineering. Fluid mechanics can be mathematically complex. Sometimes the problems can best be solved by numerical methods, typically using computers. Fluids have the ability to transport matter and its properties as well as transmit force; therefore fluid mechanics is a subject that is particularly open to cross-fertilization with other sciences and disciplines of engineering. The subject of fluid mechanics is highly relevant in such domains as mechanical, chemical, petrochemical, civil, metallurgical, biological, and ecological engineering.

The original papers explored in this special issue include a wide variety of topics such as boundary layer flows, nanofluids dynamics, heat and mass transfer, combustion theory hydromagnetic flows, gas dynamics, computational fluid dynamics, thermodynamics analysis, aerodynamics, and non-Newtonian flows. In "Travelling waves solution of the unsteady flow problem of a rarefied nonhomogeneous charged gas bounded by an oscillating plate," T. Zakaraia and A. Wahid solved the initial-boundary value problem of the Rayleigh flow applied to the system of two-component plasma (positive ions + electrons), bounded by an oscillating plate. The ratios between the different contributions of the internal energy changes are predicted via the extended Gibbs equation for both diamagnetic and paramagnetic plasmas. In "DSMC prediction of particle behavior in gas-particle two-phase impinging streams," M. Du et al. investigated the behaviour of gas-particle two-phase impinging streams
(GPISs). The collisional interaction of particles was taken into account by means of a modified direct simulation Monte Carlo (DSMC) method based on a Lagrangian approach and the modified Nanbu method. The results indicate that the particle distribution in GPIS can be divided into three zones: particle-collision zone, particle-jetting zone, and particle-scattering zone. In "Performance analysis of highspeed deep/shallow recessed hybrid bearing," L. Wang and S. Jiang presented a theoretical analysis of the performance of deep/shallow recessed hybrid bearing. By adopting bulk flow theory, the turbulent Reynolds equation and energy equation are modified and solved numerically. The results indicate that the load capacity and drag torque increase as the depth becomes shallower and the width ratio (half angle of deep recess versus half angle of shallow recess) becomes smaller. In "Feature-parameter-criterion for predicting lean blowout limit of gas turbine combustor and bluff body burner," H. Zheng et al. proposed a new method named Feature-ParameterCriterion (FPC) for predicting Lean blowout (LBO) limit using computational fluid dynamics (CFD) software FLUENT. The results show that the simulation of LBO limit based on FPC is in good agreement with the experiment data (the errors are about 5\%). In "Time-splitting procedures for the numerical solution of the $2 \mathrm{D}$ advection-diffusion equation," A. R. Appadu and H. H. Gidey presented a spectral analysis of the dispersive and dissipative properties of two timesplitting procedures, namely, locally one-dimensional (LOD) Lax-Wendroff and LOD $(1,5)$ for the numerical solution of the 
2D advection-diffusion equation. An optimization technique is implemented to find the optimal value of temporal step size that minimizes the dispersion error for both schemes when the spatial step is chosen as 0.025 . In "Model of infiltration of spent automotive catalysts by molten metal in process of platinum metals recovery," A. Fornalczyk et al. analysed the model for the washing-out process of precious metals from spent catalysts by the use of molten lead in which the metal flow is caused by the rotating electromagnetic field and the Lorentz force. The developed model enabled analysis of the impact of spacing between the catalysts and the supply current on the degree of catalyst infiltration by the molten metal. In " A fractal model for the maximum droplet diameter in gas-liquid mist flow, X. H. Tan et al. made use of fractal theory to describe liquid droplet size distribution in gasliquid mist flow. The results reveal that maximum droplet diameter decreases with an increase in gas superficial velocity but increases with an increase in liquid superficial velocity. Droplet fractal dimension also increases with an increase in gas superficial velocity but decreases with an increase in liquid superficial velocity. In "Framework for the shape optimization of aerodynamic profiles using genetic algorithms," D. López et al. developed a framework for the shape optimization of aerodynamics profiles using computational fluid dynamics (CFD) and genetic algorithms. The results obtained demonstrated the effectiveness of the developed tool. In "Nonlinear characteristics of helicopter rotor blade airfoils: an analytical evaluation", C. Rotaru examined the effect of unsteadiness on airfoil operating under nominally attached flow conditions away from stall using Theodorsen's theory, where the aerodynamic response (lift and pitching moment) is considered as a sum of noncirculatory and circulatory parts. The apparent mass contributions to the forces and pitching moments, which are proportional to the instantaneous motion, are included as part of the quasi-steady result. In "The use of fractional order derivative to predict the groundwater flow," A. N. Atangana and Bildik derived the analytical solution of the-time-fractional groundwater flow equation (TFGFE) via the Laplace-Carson transform method and the generalized Wright function. This solution obtained is in perfect agreement with the data observed from the pumping test performed by the Institute for Groundwater Study on one of its borehole settled on the test site of the University of the Free State. In "Unsteady analyses of a control valve due to fluid-structure coupling," Y. Xie et al. analysed the coupling mechanism between the structure and the fluid system at the control valve numerically. The results help understand the processes that occur in the valve flow path leading to the pressure control instability observed in the control valve in the combined cycle power plants (CCPP). In "The flow of a variable viscosity fluid down an inclined plane with a free surface," M. S. Tshehla investigated the combined effects of convective heating and temperature dependent variable viscosity on fluid flowing down an inclined plane with a free surface. The model problem is tackled numerically; graphical results are displayed and discussed quantitatively. In "A new numerical approach of MHD flow with heat and mass transfer for the UCM fluid over a stretching surface in the presence of thermal radiation," S. Shateyi and G. T. Marewo made use of spectral relaxation method in order to numerically investigate the magnetohydrodynamic boundary layer flow with heat and mass transfer of an incompressible upperconvected Maxwell fluid over a stretching sheet in the presence of viscous dissipation and thermal radiation as well as chemical reaction. The results are obtained for velocity, temperature, concentration, skin friction, and Nusselt number. In "Analytical solution of flow and heat transfer over a permeable stretching wall in a porous medium," M. Dayyan et al. investigated the boundary layer flow through a porous medium over a stretching porous wall with respect to first and second laws of thermodynamics for both isothermal and isoflux cases. It is shown that the rate of heat transfer increases with Reynolds number, Prandtl number, and suction to the surface. In "Calculation analysis of pressure wave velocity in gas and drilling mud two-phase fluid in annulus during drilling operations," Y. Lin et al. proposed a united wave velocity model based on pressure gradient equations in drilling operations, gas-liquid two-fluid model, the gas-drilling mud equations of state, and small perturbation theory. It is shown that the drop of pressure causes an increase in void fraction along the flow direction. The wave velocity tends to increase with the increase in back pressure and the decrease of gas influx rate and angular frequency, significantly in low range. In "Spectral local linearisation approach for natural convection boundary Layer flow," S. S. Motsa et al. utilised a spectral local linearisation method (SLLM) to numerically solve a model problem describing natural convection boundary layer flow with domain transformation. A comparative study between the SLLM and existing results in the literature was carried out to validate the results. The method has shown to be a promising efficient tool for nonlinear boundary value problems as it gives converging results after few iterations. In "Gel characteristics of urea-formaldehyde resin under shear flow conditions," D. Wang et al. investigated the gel characteristics of ureaformaldehyde resin (UFR) chemical grout under shear flow conditions experimentally using an apparatus which consists of a magnetic stirrer and a viscometer. The results show that the grouting flow rate influences the gelling time. Faster flow rates will cause longer gelling time, which means that the time for the grout to gel during the flowing process under shear flow conditions is longer than that under static conditions. In "On the comparison between compact finite difference and pseudospectral approaches for solving similarity boundary layer problems," P. G. Dlamini et al. presented a comparison of the compact finite difference approach against the pseudospectral approach in solving similarity boundary layer problems. It is shown that the results of the two approaches are comparable in terms of accuracy for small systems of equations. For larger systems of equations, the proposed compact finite difference approaches are more accurate than the spectral-method-based approaches. In "Radiation and magnetohydrodynamics effects on unsteady free convection flow in a porous medium," S. Ulhaq et al. examined the unsteady MHD free convection flow near an exponentially accelerated infinite vertical plate through porous medium with uniform heat flux in the presence of thermal radiation. Exact solutions are obtained by the Laplace transform method. The effects of pertinent parameters such 
as the radiation parameter, Grashof number, Prandtl number, and time on velocity, temperature, and skin friction are shown graphically and discussed quantitatively. In "CFD simulation of heat transfer and friction factor augmentation in a circular tube fitted with elliptic-cut twisted tape inserts," S. D. Salman et al. presented a numerical simulation of swirling flow in a tube induced by elliptic-cut and classical twist tape inserts using CFD package (FLUENT-6.3.26). The results show that the heat transfer rate and friction factor in the tube equipped with elliptic-cut twist tape (ECT) are significantly higher than those fitted with classical twist tape (CTT). In "Shock wave solutions for some nonlinear flow models arising in the study of a non-Newtonian third grade fluid," T. Aziz et al. presented a new class of closed-form shock wave solutions for some nonlinear problems arising in the study of a third grade fluid model using the Lie symmetry reduction technique. In "Hydromagnetic stagnation-point flow towards a radially stretching convectively heated disk," S. Shateyi and O. D. Makinde presented a numerical solution for steady MHD stagnation-point flow and heat transfer of an electrically conducted incompressible viscous fluid over a convectively heat and radially stretching disk surface. Pertinent results on the effects of various thermophysical parameters on the velocity and temperature fields as well as local skin friction and local Nusselt number are discussed in detail and shown graphically and/or in tabular form. In "MHD boundary layer flow due to exponential stretching surface with radiation and chemical reaction," Y. I Seini and O. D. Makinde investigated the combined effects of thermal radiation and first order homogeneous chemical reaction on hydromagnetic boundary layer flow of a viscous, steady, and incompressible fluid over an exponential stretching sheet. It was found that the rate of heat transfer at the surface decreases with increasing values of the transverse magnetic field parameter and the radiation parameter. In "Entropy generation analysis in a variable viscosity MHD channel flow with permeable walls and convective heating," A. S. Eegunjobi and O. D. Makinde numerically analysed inherent irreversibility in a steady flow of an incompressible variable viscosity electrically conducting fluid through a channel with permeable walls and convective surface boundary conditions. The results revealed that entropy generation minimization can be achieved by appropriate combination of the regulated values of thermophysical parameters controlling the flow systems. In "Boundary layer flow past a wedge moving in a nanofluid," W. A. Khan and I. Pop numerically studied the problem of steady boundary layer flow of nanofluid past a stretching wedge with a parallel free stream velocity. It is found that the velocity at the surface increases/decreases with stretching/shrinking parameters while the temperature increases with both Brownian motion and thermophoresis parameters.

Thus, the papers in this special issue, representing a broad spectrum of both theoretical and experimental approaches and areas of investigation, demonstrate the wide array of new development in fluid mechanics and its engineering applications. We are certain that the papers published in this special issue will contribute to advancement of knowledge in the field of fluid mechanics and provide scientists, engineers, industries, research scholars, and practitioners' latest theoretical and technological achievements in fluid mechanics and its various engineering applications.

\section{Acknowledgments}

We would like to thank the authors for their contribution and the reviewers for their collaboration.

Oluwole Daniel Makinde Waqar Ahmed Khan Tirivanhu Chinyoka 


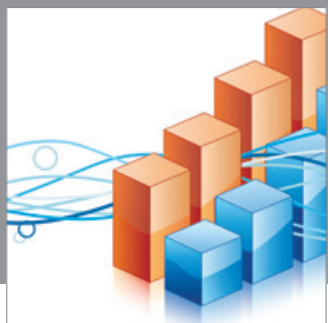

Advances in

Operations Research

mansans

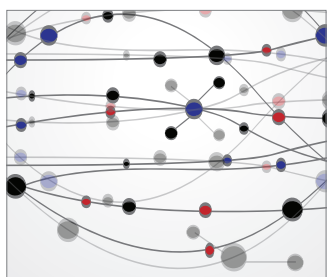

The Scientific World Journal
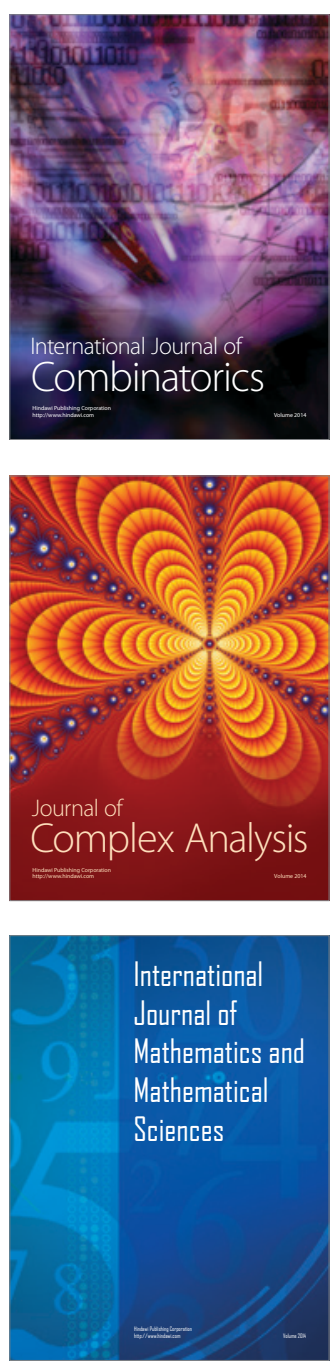
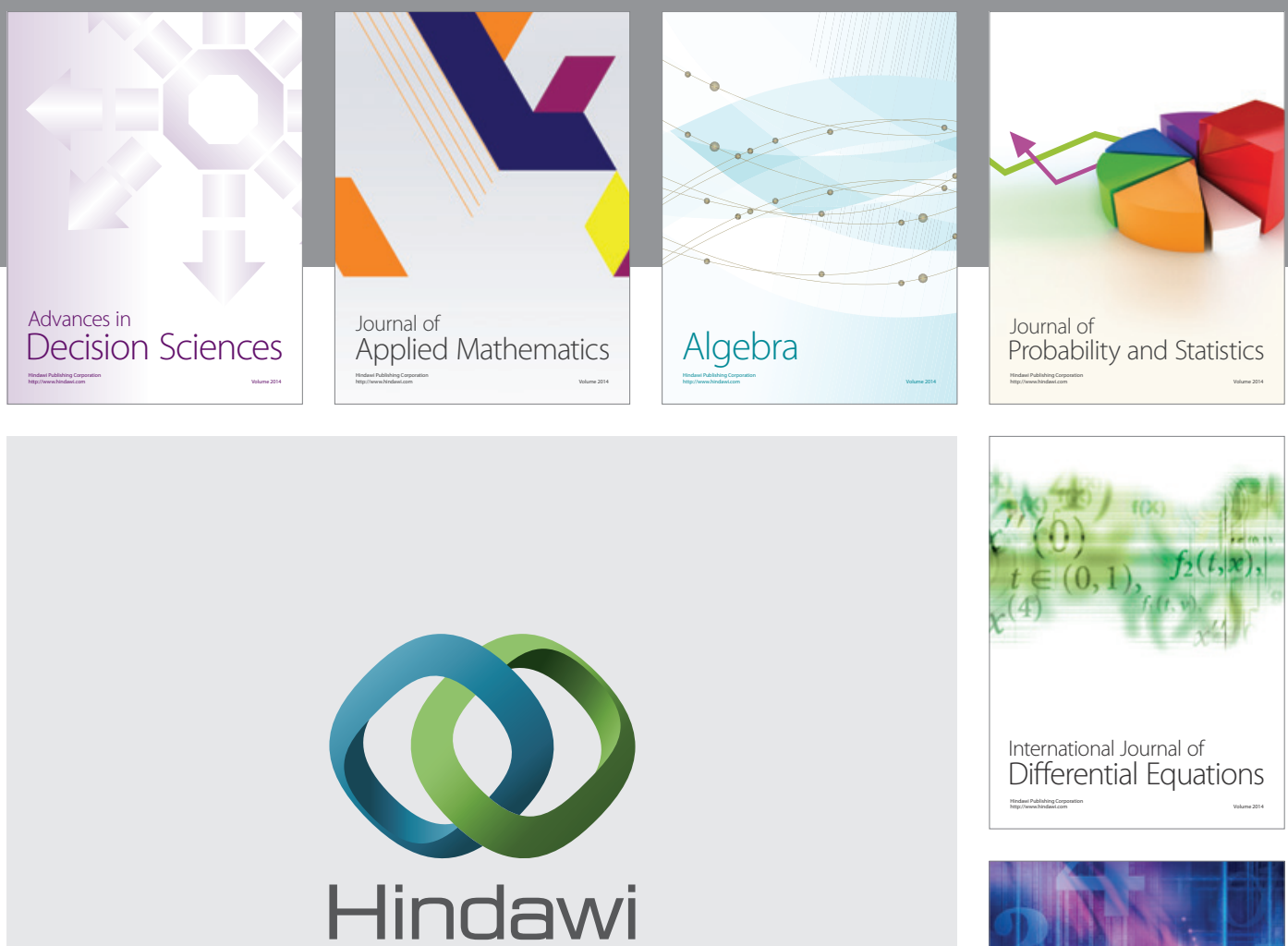

Submit your manuscripts at http://www.hindawi.com
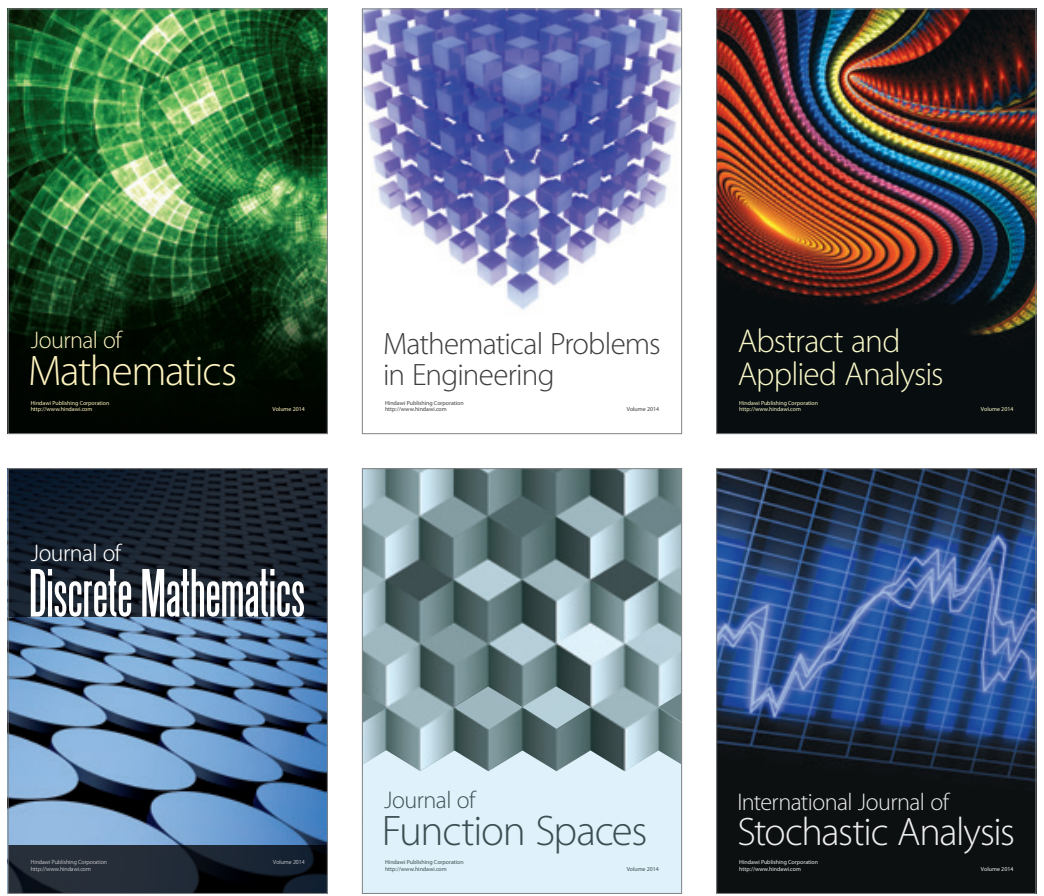

Journal of

Function Spaces

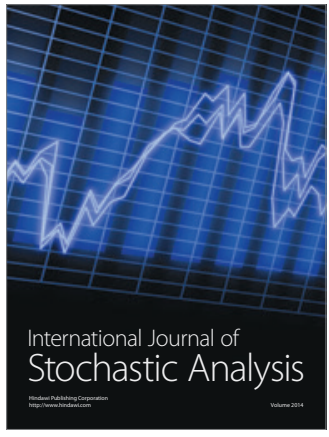

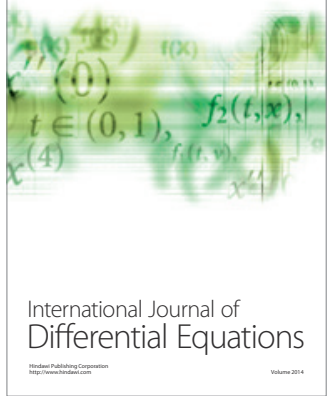
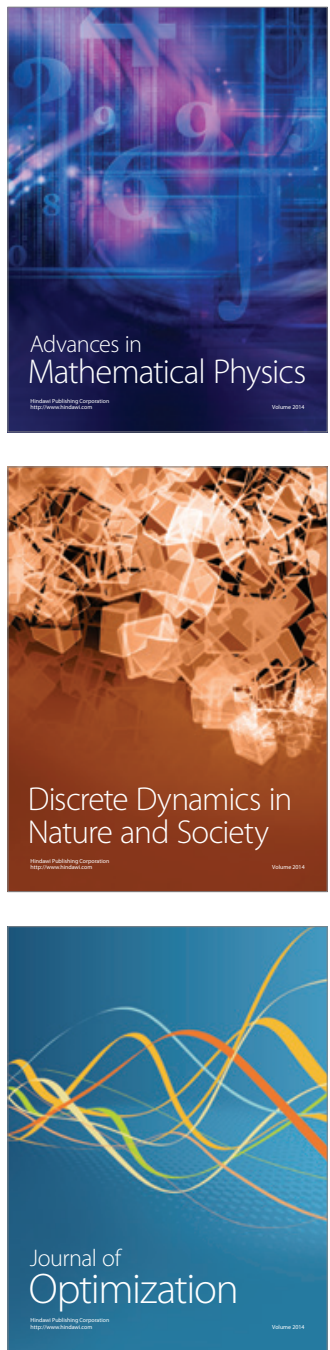